- Supporting Information -

\title{
2-Methoxyethylamino-bis(phenolate)yttrium Catalysts for the Synthesis of Highly Isotactic Poly(2-vinylpyridine) by Rare-Earth Metal-Mediated Group Transfer Polymerization
}

Alexander Kronast, ${ }^{\ddagger}$ Dominik Reiter, ${ }^{\ddagger}$ Peter T. Altenbuchner, Sergei I. Vagin, and Bernhard Rieger*

WACKER-Lehrstuhl für Makromolekulare Chemie, Technische Universität München, Lichtenbergstraße 4, 85748 Garching b. München, Germany.

*B. Rieger: E-Mail: rieger@tum.de; http://www.makro.ch.tum.de; Fax: +49-89-289-13562; Tel: +49-89-289-13570.

1. Experimental 1

1.1 General 1

1.2 Proligand Precursor Synthesis 2

2. Kinetic Investigations 6

3. Microstructure Analysis $\quad 7$

$3.1 \quad{ }^{13} \mathrm{C}$ NMR spectra $\quad 7$

3.2 Theoretical Investigations 10

4. Thermoanalysis 11

4.1 DSC 11

$\begin{array}{lll}4.2 \text { TGA } & 12\end{array}$

5. Literature 13 


\section{Experimental}

\subsection{General}

All air and moisture sensitive reactions were carried out under an argon atmosphere using standard Schlenk or glovebox techniques. All glassware was heat dried under vacuum prior to use. Unless otherwise stated, all chemicals were purchased from Acros Organics, Sigma-Aldrich, or ABCR and used as received. Dichloromethane, pentane, THF and toluene were dried using an MBraun SPS-800 solvent purification system. The precursor complex $\left[\mathrm{Y}\left(\mathrm{CH}_{2} \mathrm{SiMe}_{3}\right)_{3}(\mathrm{THF})_{2}\right]$ was prepared following literature procedures. ${ }^{[1]}$ Triethylamine and 2-vinylpyridine were dried over calcium hydride and distilled prior to use. 2-(((2-methoxyethyl)amino)methyl)-4,6-dimethylphenol and 2,4-di-tert-butyl-6-(((2-methoxyethyl)amino)methyl)phenol were synthesized according to literature procedures. ${ }^{[2]}$

NMR Spectra were recorded on Bruker AVIII-300 and AVIII-500C spectrometers. ${ }^{1} \mathrm{H}$ NMR spectroscopic chemical shifts $\delta$ are reported in ppm relative to the residual proton signals of deuterated solvents. Unless otherwise stated ${ }^{13} \mathrm{C}$ NMR spectroscopic chemical shifts $\delta$ are referenced to the carbon atoms of the solvent. Coupling constants $J$ are given in Hertz $(\mathrm{Hz})$ as averaged values and refer to couplings between protons. The following abbreviations are used to describe signal multiplicities: $\mathrm{s}$ (singlet), $\mathrm{d}$ (doublet), $\mathrm{t}$ (triplet), $\mathrm{m}$ (multiplet), dd (doublet of doublets), $\mathrm{dt}$ (doublet of triplets), br (broad signal). Deuterated solvents were obtained from Sigma-Aldrich and dried over $3 \AA$ molecular sieves.

Quantitative elemental analyses (EA) were measured at the Laboratory for Microanalysis at the Institute of Inorganic Chemistry at the Technische Universität München. Low resolution mass spectrometry (LRMS) analytical measurements were obtained by electrospray ionization (ESI) with acetonitrile and toluene solutions on a Varian LC/MS 500-MS spectrometer. X-Ray Powder Diffraction was measured on a PANalytical Empyrean system. DSC was carried out on a Texas Instruments DSC Q2000 with a heating rate of $5 \mathrm{~K} \mathrm{~min}^{-1}$. TGA was carried out on a Texas Instruments TGA Q5000 with a heating rate of $10 \mathrm{~K} \mathrm{~min}^{-1}$. Gel permeation chromatography (GPC) measurements were carried out on a Varian 920-LC HPLC equipped with two PL Polargel columns.

Initiator efficiency $\left(I^{*}=\mathrm{M}_{\mathrm{n} \text {,calc }} / \mathrm{M}_{\mathrm{n} \text {,exp }}\right)$ is determined by taking aliquots during and at the end of the polymerization. $I^{*}$ is determined for polymerization kinetics as the average initiator efficiency $I^{*}$ at the maximum rate of the reaction (maximum slope of the conversion-reaction time plot). 


\subsection{Proligand Precursor Synthesis}
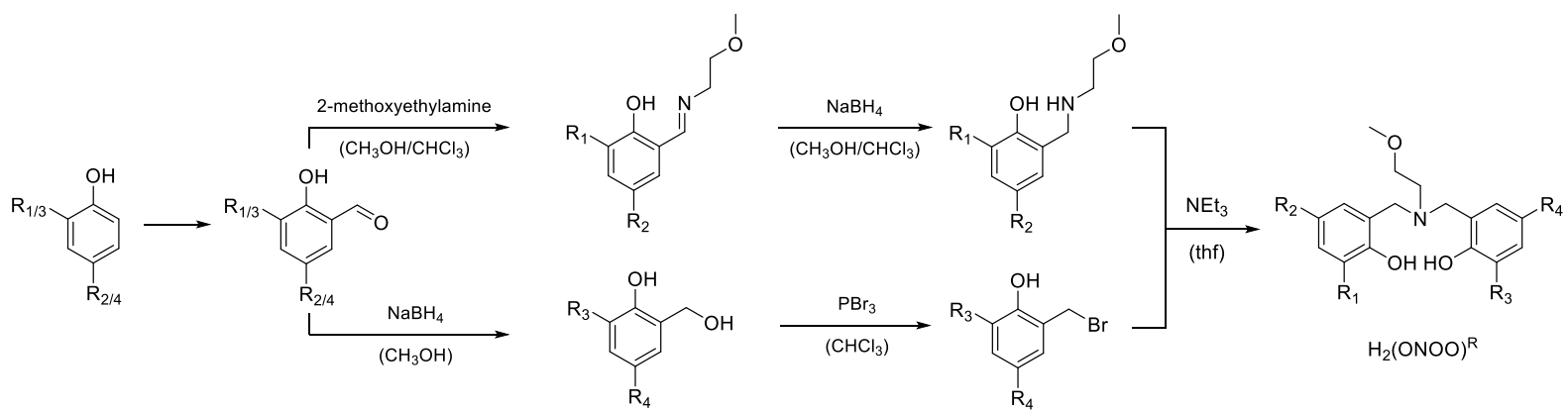

Scheme S1. Proligand synthesis starting from phenols 3 and 10 for Ligands L1-L4.

\section{Tris(4-tert-butylphenyl)methanol (1)}

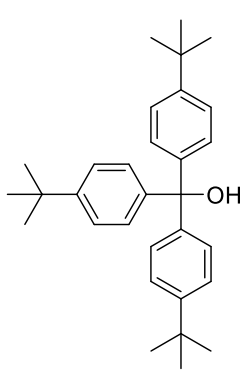

$\mathrm{C}_{31} \mathrm{H}_{40} \mathrm{O}$

$428.65 \mathrm{~g} / \mathrm{mol}$

A solution of 1-bromo-4-tert-butylbenzene $(24.4 \mathrm{~mL}, 30.0 \mathrm{~g}, 141 \mathrm{mmol}, 3.0 \mathrm{eq})$ in dry THF $(20 \mathrm{~mL})$ was added dropwise to a stirred suspension of magnesium turnings $(3.76 \mathrm{~g}, 155 \mathrm{mmol}$, $3.3 \mathrm{eq})$ in dry THF $(200 \mathrm{~mL})$. The reaction mixture was heated to initiate the reaction and stirred until the reaction subsided. A solution of diethyl carbonate $(5.69 \mathrm{~mL}, 5.54 \mathrm{~g}, 46.9 \mathrm{mmol}$, $1.0 \mathrm{eq})$ in dry THF $(100 \mathrm{~mL})$ was added dropwise to the Grignard reagent and stirred for $3 \mathrm{~h}$ at room temperature. Ice-cold $2 \mathrm{M} \mathrm{HCl}(100 \mathrm{~mL})$ was then poured into the reaction mixture and the organic phase was separated and washed with $2 \mathrm{M} \mathrm{HCl}(50 \mathrm{~mL})$ and brine $(50 \mathrm{~mL})$. The organic phase was dried over $\mathrm{MgSO}_{4}$, filtered and the solution concentrated in vacuo. Addition of $n$-hexane led to precipitation of $\mathbf{1}$ as colorless crystals (12.4 g, $29.0 \mathrm{mmol}, 62 \%)$.

${ }^{1} \mathrm{H}$ NMR $\left(300 \mathrm{MHz}, \mathrm{CDCl}_{3}, 300 \mathrm{~K}\right): \delta[\mathrm{ppm}]=1.31\left(\mathrm{~s}, 27 \mathrm{H}, \mathrm{C}\left(\mathrm{CH}_{3}\right)_{3}\right), 7.19\left(\mathrm{~d},{ }^{3} J=8.5 \mathrm{~Hz}\right.$, $6 \mathrm{H}, \mathrm{Ar}-\mathrm{H}), 7.31\left(\mathrm{~d},{ }^{3} \mathrm{~J}=8.5 \mathrm{~Hz}, 6 \mathrm{H}, \mathrm{Ar}-\mathrm{H}\right)$.

${ }^{13} \mathrm{C}$ NMR $\left(75 \mathrm{MHz}, \mathrm{CDCl}_{3}, 300 \mathrm{~K}\right): \delta[\mathrm{ppm}]=31.5,34.6,81.7,124.8,127.7,144.3,149.9$.

Tris(4-tert-butylphenyl)methyl chloride (2)

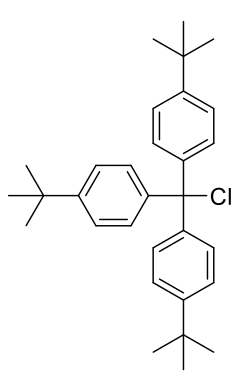

To a vigorously stirred solution of $1(12.4 \mathrm{~g}, 29.0 \mathrm{mmol}, 1.0 \mathrm{eq})$ in dry toluene $(30 \mathrm{~mL})$ was added freshly distilled acetyl chloride $(5.18 \mathrm{~mL}, 5.69 \mathrm{~g}, 72.6 \mathrm{mmol}, 2.5 \mathrm{eq})$. The reaction mixture was heated under reflux for $30 \mathrm{~min}$. After the solution was allowed to cool down to ambient temperature, $n$-hexane $(40 \mathrm{~mL})$ was added and the flask was left at $4{ }^{\circ} \mathrm{C}$ overnight. The resulting precipitate was filtered and dried under vacuum to afford 2 as a beige powder $(10.0 \mathrm{~g}$, $22.4 \mathrm{mmol}, 77 \%)$.

${ }^{1} \mathrm{H}$ NMR $\left(300 \mathrm{MHz}, \mathrm{CDCl}_{3}, 300 \mathrm{~K}\right): \delta[\mathrm{ppm}]=1.33\left(\mathrm{~s}, 27 \mathrm{H}, \mathrm{C}\left(\mathrm{CH}_{3}\right)_{3}\right), 7.16\left(\mathrm{~d},{ }^{3} J=8.6 \mathrm{~Hz}, 6 \mathrm{H}\right.$, Ar-H), $7.31\left(\mathrm{~d},{ }^{3} \mathrm{~J}=8.6 \mathrm{~Hz}, 6 \mathrm{H}, \mathrm{Ar}-\mathrm{H}\right)$.

${ }^{13} \mathrm{C}$ NMR $\left(75 \mathrm{MHz}, \mathrm{CDCl}_{3}, 300 \mathrm{~K}\right): \delta[\mathrm{ppm}]=31.5,34.7,81.8,124.6,129.5,142.7,150.6$.

$\mathrm{C}_{31} \mathrm{H}_{39} \mathrm{Cl}$

$447.09 \mathrm{~g} / \mathrm{mol}$

\section{4-tert-Butyl-2-(tris(4-tert-butylphenyl)methyl)phenol (3)}

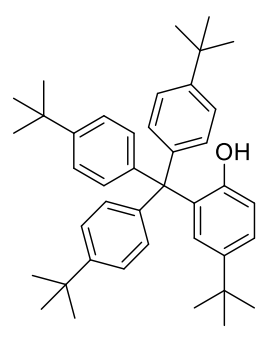

$\mathrm{C}_{41} \mathrm{H}_{52} \mathrm{O}$

$560.85 \mathrm{~g} / \mathrm{mol}$

To molten 4-tert-butylphenol (33.7 g, $224 \mathrm{mmol}, 10.0 \mathrm{eq})$ was added sodium metal (722 mg, $31.4 \mathrm{mmol}, 1.4 \mathrm{eq})$ at $110^{\circ} \mathrm{C}$ with vigorous stirring. $2(10.0 \mathrm{~g}, 22.4 \mathrm{mmol}, 1.0 \mathrm{eq})$ was added to the melt of phenolate and the resulting mixture was heated to $145{ }^{\circ} \mathrm{C}$ for $3 \mathrm{~h}$. The reaction mixture was treated with $7 \%$ aqueous $\mathrm{NaOH}(67 \mathrm{~mL})$ and $\mathrm{Et}_{2} \mathrm{O}(77 \mathrm{~mL})$ after cooling down to $80^{\circ} \mathrm{C}$. The organic layer was separated and the aqueous phase extracted with $\mathrm{Et}_{2} \mathrm{O}(3 \times 34 \mathrm{~mL})$. The combined organic phases were washed with $7 \%$ aqueous $\mathrm{NaOH}(3 \times 34 \mathrm{~mL})$, distilled water $(34 \mathrm{~mL})$ and brine $(34 \mathrm{~mL})$. After drying over anhydrous $\mathrm{MgSO}_{4}$ and filtration, the solvent of the amber solution was removed in vacuo. Recrystallization of the crude product from ethanol yielded 3 as colorless crystals (9.99 g, $17.8 \mathrm{mmol}, 79 \%)$.

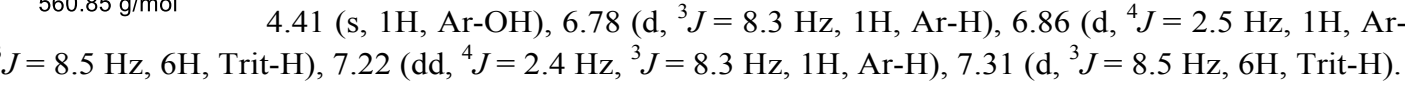

${ }^{13} \mathrm{C}$ NMR $\left(126 \mathrm{MHz}, \mathrm{CDCl}_{3}, 300 \mathrm{~K}\right): \delta[\mathrm{ppm}]=31.5,31.5,34.3,34.5,61.8,117.0,124.7,125.3,128.1,130.8$, $133.0,141.3,142.5,149.5,152.2$.

LRMS (ESI, MeCN): $\mathrm{m} / \mathrm{z}=584.2\left[(\mathrm{M}+\mathrm{Na})^{+}\right]$.

EA [\%]: calculated: C $87.80 \mathrm{H} 9.35$; found: C $87.94 \mathrm{H} 9.43$ 


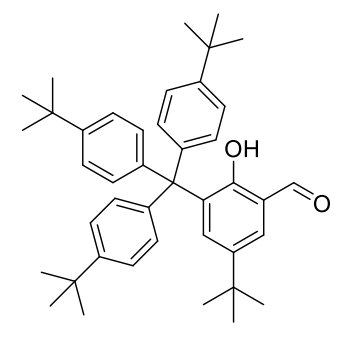

$\mathrm{C}_{42} \mathrm{H}_{52} \mathrm{O}_{2}$ $588.86 \mathrm{~g} / \mathrm{mol}$

A mixture of 3 (9.96 g, $17.8 \mathrm{mmol}, 1.0 \mathrm{eq})$, hexamethylenetetramine $(2.74 \mathrm{~g}, 19.5 \mathrm{mmol}$, $1.1 \mathrm{eq})$ and trifluoroacetic acid $(100 \mathrm{~mL})$ was heated at $110{ }^{\circ} \mathrm{C}$ under reflux for $40 \mathrm{~h}$. Subsequently the warm reaction mixture was poured into vigorously stirred $2 \mathrm{M} \mathrm{HCl}$ $(250 \mathrm{~mL})$. The cold mixture was extracted with chloroform $(3 \times 125 \mathrm{~mL})$ and the combined organic layers were washed with $2 \mathrm{M} \mathrm{HCl}(3 \times 75 \mathrm{~mL})$, distilled water $(175 \mathrm{~mL})$ and brine $(100 \mathrm{~mL})$. After drying over $\mathrm{MgSO}_{4}$ and filtration, the solvent was removed under reduced pressure. Silica gel column chromatography ( $n$-hexane:EtOAc $=30: 1)$ was utilized to purify the residue and afford 4 as a white powder $(6.65 \mathrm{~g}, 11.3 \mathrm{mmol}, 64 \%)$.

TLC: $R_{\mathrm{f}}=0.19$ (silica gel, $n$-hexane:EtOAc $=30: 1$, [UV]).

${ }^{1} \mathrm{H}$ NMR $\left(500 \mathrm{MHz}, \mathrm{CDCl}_{3}, 300 \mathrm{~K}\right): \delta[\mathrm{ppm}]=1.16\left(\mathrm{~s}, 9 \mathrm{H}, \mathrm{C}\left(\mathrm{CH}_{3}\right)_{3}\right), 1.31(\mathrm{~s}, 27 \mathrm{H}$, $\left.\mathrm{C}\left(\mathrm{CH}_{3}\right)_{3}\right), 7.09\left(\mathrm{~d},{ }^{3} J=8.6 \mathrm{~Hz}, 6 \mathrm{H}\right.$, Trit-H), $7.25\left(\mathrm{~d},{ }^{3} J=8.5 \mathrm{~Hz}, 6 \mathrm{H}\right.$, Trit-H), $7.33\left(\mathrm{~d},{ }^{4} J=\right.$ $2.5 \mathrm{~Hz}, 1 \mathrm{H}, \mathrm{Ar}-\mathrm{H}), 7.43$ (d, $\left.{ }^{4} J=2.5 \mathrm{~Hz}, 1 \mathrm{H}, \mathrm{Ar}-\mathrm{H}\right), 9.86$ (s, 1H, CHO), 11.13 (s, 1H, Ar-OH).

${ }^{13} \mathrm{C}$ NMR $\left(126 \mathrm{MHz}, \mathrm{CDCl}_{3}, 300 \mathrm{~K}\right): \delta[\mathrm{ppm}]=31.2,31.5,34.3,34.4,62.1,120.2,124.1,128.8,130.6,136.0$, $136.7,141.4,142.1,148.5,158.8,197.0$.

LRMS (ESI, MeCN): $\mathrm{m} / \mathrm{z}=612.2\left[(\mathrm{M}+\mathrm{Na})^{+}\right]$.

EA [\%]: calculated: C 85.67, H 8.90; found: C 85.59, H 9.05.

\section{4-tert-Butyl-2-hydroxymethyl-6-(tris(4-tert-butylphenyl)methyl)phenol (5)}

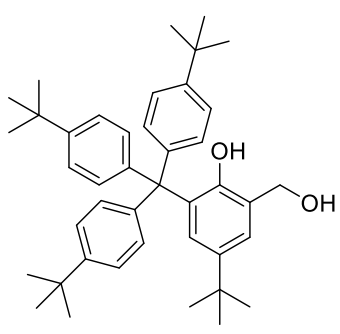

$\mathrm{C}_{42} \mathrm{H}_{54} \mathrm{O}_{2}$ $590.88 \mathrm{~g} / \mathrm{mol}$

To a suspension of $4(3.10 \mathrm{~g}, 5.26 \mathrm{mmol}, 1.0 \mathrm{eq})$ in methanol $(100 \mathrm{~mL})$ was added sodium borohydride (398 mg, $10.5 \mathrm{mmol}, 2.0 \mathrm{eq}$ ) in small portions. After the reaction mixture was stirred at room temperature for $1 \mathrm{~h}$, the solvent was removed under reduced pressure. The residue was redissolved in distilled water $(150 \mathrm{~mL})$ and neutralized with glacial acetic acid. The aqueous phase was extracted with chloroform $(3 \times 100 \mathrm{~mL})$ and the combined organic layers were dried over anhydrous $\mathrm{MgSO}_{4}$. After filtration, the solvent was removed in vacuo to yield 6 as a white powder (3.09 g, $5.23 \mathrm{mmol}, 99 \%)$. The product was utilized in the next step without further purification.

${ }^{1} \mathrm{H}$ NMR $\left(500 \mathrm{MHz}, \mathrm{CDCl}_{3}, 300 \mathrm{~K}\right): \delta[\mathrm{ppm}]=1.11\left(\mathrm{~s}, 9 \mathrm{H}, \mathrm{C}\left(\mathrm{CH}_{3}\right)_{3}\right), 1.31(\mathrm{~s}, 27 \mathrm{H}$, $\left.\mathrm{C}\left(\mathrm{CH}_{3}\right)_{3}\right), 2.45$ (br s, $\left.1 \mathrm{H}, \mathrm{CH}_{2} \mathrm{OH}\right), 4.64\left(\mathrm{~s}, 2 \mathrm{H}, \mathrm{CH}_{2}\right), 4.86(\mathrm{~s}, 1 \mathrm{H}, \mathrm{Ar}-\mathrm{OH}), 6.84\left(\mathrm{~d},{ }^{4} \mathrm{~J}=\right.$ $7.30\left(\mathrm{~d},{ }^{3} \mathrm{~J}=8.5 \mathrm{~Hz}, 6 \mathrm{H}\right.$, Trit-H).

$2.4 \mathrm{~Hz}, 1 \mathrm{H}, \mathrm{Ar}-\mathrm{H}), 7.11\left(\mathrm{~d},{ }^{3} J=8.5 \mathrm{~Hz}, 6 \mathrm{H}\right.$, Trit-H), $7.19\left(\mathrm{~d},{ }^{4} J=2.4 \mathrm{~Hz}, 1 \mathrm{H}, \mathrm{Ar}-\mathrm{H}\right)$,

${ }^{13} \mathrm{C}$ NMR $\left(126 \mathrm{MHz}, \mathrm{CDCl}_{3}, 300 \mathrm{~K}\right): \delta[\mathrm{ppm}]=31.5,31.5,34.3,34.5,61.8,63.5,124.7,125.0,127.8,127.9,130.7$, $133.2,141.3,142.2,149.5,150.6$.

LRMS (ESI, MeCN): $\mathrm{m} / \mathrm{z}=614.2\left[(\mathrm{M}+\mathrm{Na})^{+}\right]$.

EA [\%]: calculated: C 85.37, H 9.21; found: C 79.31, H 8.63.

\section{2-Bromomethyl-4-tert-butyl-6-(tris(4-tert-butylphenyl)methyl)phenol (6)}

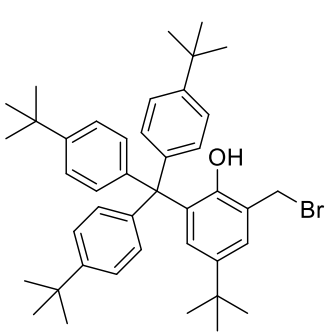

$\mathrm{C}_{42} \mathrm{H}_{53} \mathrm{BrO}$ $653.77 \mathrm{~g} / \mathrm{mol}$

To a solution of 5 (3.06 g, $5.18 \mathrm{mmol}, 1.0 \mathrm{eq})$ in chloroform $(100 \mathrm{~mL})$ was added phosphorus tribromide $(0.25 \mathrm{~mL}, 701 \mathrm{mg}, 2.59 \mathrm{mmol}, 0.5 \mathrm{eq})$. After the reaction mixture was stirred at room temperature for $1 \mathrm{~h}$, iced water $(40 \mathrm{~mL})$ was added over $5 \mathrm{~min}$. The resulting phases were separated and the aqueous phase was extracted with chloroform (3 $\times 25 \mathrm{~mL}$ ). The combined organic layers were dried over anhydrous $\mathrm{MgSO}_{4}$, filtered and the solvent removed in vacuo to afford 7 as a white powder $(3.39 \mathrm{~g}, 5.18 \mathrm{mmol}$, quantitative yield).

${ }^{1} \mathrm{H}$ NMR $\left(500 \mathrm{MHz}, \mathrm{CDCl}_{3}, 300 \mathrm{~K}\right): \delta[\mathrm{ppm}]=1.11\left(\mathrm{~s}, 9 \mathrm{H}, \mathrm{C}\left(\mathrm{CH}_{3}\right)_{3}\right), 1.31(\mathrm{~s}, 27 \mathrm{H}$, $\left.\mathrm{C}\left(\mathrm{CH}_{3}\right)_{3}\right), 4.53\left(\mathrm{~s}, 2 \mathrm{H}, \mathrm{CH}_{2}\right), 4.77(\mathrm{~s}, 1 \mathrm{H}, \mathrm{Ar}-\mathrm{OH}), 6.87\left(\mathrm{~d},{ }^{4} J=2.4 \mathrm{~Hz}, 1 \mathrm{H}, \mathrm{Ar}-\mathrm{H}\right), 7.10$ $\left(\mathrm{d},{ }^{3} J=8.6 \mathrm{~Hz}, 6 \mathrm{H}\right.$, Trit-H), $7.25\left(\mathrm{~d},{ }^{4} J=3.0 \mathrm{~Hz}, 1 \mathrm{H}, \mathrm{Ar}-\mathrm{H}\right), 7.30\left(\mathrm{~d},{ }^{3} J=8.6 \mathrm{~Hz}, 6 \mathrm{H}\right.$, Trit-H).

${ }^{13} \mathrm{C}$ NMR $\left(126 \mathrm{MHz}, \mathrm{CDCl}_{3}, 300 \mathrm{~K}\right): \delta[\mathrm{ppm}]=31.1,31.4,31.5,34.3,34.5,61.9,124.3,124.8,126.6,129.2,130.7$, 133.7, 141.0, 142.4, 149.7, 150.7 .

LRMS (ESI, MeCN): m/z=677.1 [(M+Na $\left.)^{+}\right]$. 
Tris(3,5-dimethylphenyl)methanol (7)

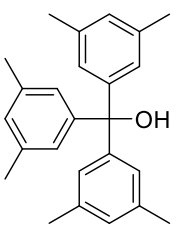

$\mathrm{C}_{25} \mathrm{H}_{28} \mathrm{O}$

$344.49 \mathrm{~g} / \mathrm{mol}$

A solution of 1-bromo-3,5-dimethylbenzene (18.4 mL, $25.0 \mathrm{~g}, 135 \mathrm{mmol}, 3.0 \mathrm{eq})$ in dry THF (20 $\mathrm{mL}$ ) was added dropwise to a stirred suspension of magnesium turnings ( $3.61 \mathrm{~g}, 149 \mathrm{mmol}, 3.3 \mathrm{eq}$ ) in dry THF $(200 \mathrm{~mL})$. The reaction mixture was heated to initiate the reaction and stirred until the reaction subsided. A solution of diethyl carbonate $(5.46 \mathrm{~mL}, 5.32 \mathrm{~g}, 45.0 \mathrm{mmol}, 1.0 \mathrm{eq})$ in dry THF $(100 \mathrm{~mL})$ was added dropwise to the Grignard reagent and stirred for $3 \mathrm{~h}$ at room temperature. Icecold $2 \mathrm{M} \mathrm{HCl}(100 \mathrm{~mL})$ was then poured into the reaction mixture and the organic phase was separated and washed with $2 \mathrm{M} \mathrm{HCl}(50 \mathrm{~mL})$ and brine $(50 \mathrm{~mL})$. The organic phase was dried over $\mathrm{MgSO}_{4}$, filtered and the solution concentrated in vacuo. Addition of $n$-hexane led to precipitation of 8 as colorless crystals $(7.96 \mathrm{~g}, 23.1 \mathrm{mmol}, 51 \%)$.

${ }^{1} \mathrm{H} \mathrm{NMR}\left(300 \mathrm{MHz}, \mathrm{CDCl}_{3}, 300 \mathrm{~K}\right): \delta[\mathrm{ppm}]=2.27\left(\mathrm{~s}, 18 \mathrm{H}, \mathrm{CH}_{3}\right), 2.68(\mathrm{~s}, 1 \mathrm{H}, \mathrm{OH}), 6.88(\mathrm{~s}, 6 \mathrm{H}, \mathrm{Ar}-\mathrm{H}), 6.91(\mathrm{~s}$, $3 \mathrm{H}, \mathrm{Ar}-\mathrm{H})$.

${ }^{13} \mathrm{C} \mathrm{NMR}\left(75 \mathrm{MHz}, \mathrm{CDCl}_{3}, 300 \mathrm{~K}\right): \delta[\mathrm{ppm}]=21.7,83.2,126.7,128.6,136.5,143.7$.

\section{Tris(3,5-dimethylphenyl)methyl chloride (8)}

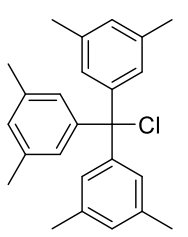

To a vigorously stirred solution of $7(7.96 \mathrm{~g}, 23.1 \mathrm{mmol}, 1.0 \mathrm{eq})$ in dry toluene $(25 \mathrm{~mL})$ was added freshly distilled acetyl chloride $(4.12 \mathrm{~mL}, 4.54 \mathrm{~g}, 57.8 \mathrm{mmol}, 2.5 \mathrm{eq})$. The reaction mixture was heated under reflux for $30 \mathrm{~min}$. After the solution was allowed to cool down to ambient temperature, $n$-hexane $(35 \mathrm{~mL})$ was added and the flask was left at $4{ }^{\circ} \mathrm{C}$ overnight. The resulting precipitate was filtered and dried under vacuum to furnish 9 as colorless crystals $(6.44 \mathrm{~g}, 17.7 \mathrm{mmol}, 77 \%)$.

${ }^{1} \mathrm{H}$ NMR $\left(500 \mathrm{MHz}, \mathrm{CDCl}_{3}, 300 \mathrm{~K}\right): \delta[\mathrm{ppm}]=2.29\left(\mathrm{~s}, 18 \mathrm{H}, \mathrm{CH}_{3}\right), 6.88(\mathrm{~s}, 6 \mathrm{H}, \mathrm{Ar}-\mathrm{H}), 6.95(\mathrm{~s}, 3 \mathrm{H}$,

$\mathrm{C}_{25} \mathrm{H}_{27} \mathrm{Cl}$ Ar-H).

$362.93 \mathrm{~g} / \mathrm{mol} \quad{ }^{13} \mathrm{C} \mathrm{NMR}\left(126 \mathrm{MHz}, \mathrm{CDCl}_{3}, 300 \mathrm{~K}\right): \delta[\mathrm{ppm}]=21.6,82.2,127.7,129.5,137.1,145.6$.

EA [\%]: calculated: C 82.73, H 7.50, Cl 9.77; found: C 82.23, H 7.45, Cl 9.30

\section{4-tert-Butyl-2-(tris(3,5-dimethylphenyl)methyl)phenol (9)}



$\mathrm{C}_{35} \mathrm{H}_{40} \mathrm{O}$

$476.69 \mathrm{~g} / \mathrm{mol}$

To molten 4-tert-butylphenol (25.7 g, $171 \mathrm{mmol}, 10.0 \mathrm{eq})$ was added sodium metal (550 mg, 23.9 mmol, $1.4 \mathrm{eq})$ at $110^{\circ} \mathrm{C}$ with vigorous stirring. $8(6.20 \mathrm{~g}, 17.1 \mathrm{mmol}, 1.0 \mathrm{eq})$ was added to the formed phenolate and the resulting mixture was heated at $145^{\circ} \mathrm{C}$ under reflux for $3 \mathrm{~h}$. The reaction mixture was treated with $7 \%$ aqueous $\mathrm{NaOH}(52 \mathrm{~mL})$ and $\mathrm{Et}_{2} \mathrm{O}(60 \mathrm{~mL})$ after cooling down to about $80^{\circ} \mathrm{C}$. The organic layer was separated and the aqueous phase extracted with $\mathrm{Et}_{2} \mathrm{O}$ $(3 \times 26 \mathrm{~mL})$. The combined organic phases were washed with $7 \%$ aqueous $\mathrm{NaOH}(3 \times 26 \mathrm{~mL})$, distilled water $(26 \mathrm{~mL})$ and brine $(26 \mathrm{~mL})$. After drying over anhydrous $\mathrm{MgSO}_{4}$ and filtration, the solvent of the amber solution was removed in vacuo. Recrystallization of the crude product from ethanol yielded $\mathbf{1 0}$ as colorless crystals $(5.72 \mathrm{~g}, 12.0 \mathrm{mmol}, 70 \%)$.

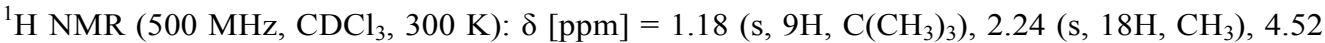
(s, $1 \mathrm{H}, \mathrm{Ar}-\mathrm{OH}), 6.73\left(\mathrm{~d},{ }^{3} J=8.2 \mathrm{~Hz}, 1 \mathrm{H}, \mathrm{Ar}-\mathrm{H}\right), 6.81\left(\mathrm{~s}, 6 \mathrm{H}\right.$, Trit-H), $6.86\left(\mathrm{~s}, 3 \mathrm{H}\right.$, Trit-H), $7.16\left(\mathrm{~d},{ }^{4} J=2.4 \mathrm{~Hz}, 1 \mathrm{H}\right.$, Ar-H), $7.20\left(\mathrm{dd},{ }^{4} \mathrm{~J}=2.4 \mathrm{~Hz},{ }^{3} \mathrm{~J}=8.3 \mathrm{~Hz}, 1 \mathrm{H}, \mathrm{Ar}-\mathrm{H}\right)$.

${ }^{13} \mathrm{C} \mathrm{NMR}\left(126 \mathrm{MHz}, \mathrm{CDCl}_{3}, 300 \mathrm{~K}\right): \delta[\mathrm{ppm}]=21.8,31.6,34.3,62.7,117.1,125.0,128.3,128.3,128.9,132.6$, 137.0, 142.2, 144.6, 152.4.

LRMS (ESI, MeCN): $\mathrm{m} / \mathrm{z}=500.2\left[(\mathrm{M}+\mathrm{Na})^{+}\right]$.

EA [\%]: calculated: C 88.19, H 8.46; found: C 88.09, H 8.57. 


\section{5-tert-Butyl-2-hydroxy-3-(tris(3,5-dimethylphenyl)methyl)benzaldehyde (10)}

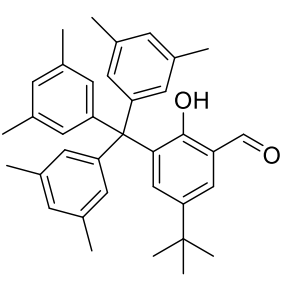

$\mathrm{C}_{36} \mathrm{H}_{40} \mathrm{O}_{2}$ $504.70 \mathrm{~g} / \mathrm{mol}$

To a suspension of $9(6.00 \mathrm{~g}, 12.6 \mathrm{mmol}, 1.0 \mathrm{eq})$, anhydrous magnesium chloride $(2.64 \mathrm{~g}$, $27.7 \mathrm{mmol}, 2.2 \mathrm{eq})$ and paraformaldehyde $(831 \mathrm{mg}, 27.7 \mathrm{mmol}, 2.2 \mathrm{eq})$ in dry THF $(70 \mathrm{~mL})$ was added dropwise freshly distilled triethylamine $(1.76 \mathrm{~mL}, 1.27 \mathrm{~g}, 12.6 \mathrm{mmol}, 1.0 \mathrm{eq})$. The resulting reaction mixture was heated under reflux for $3 \mathrm{~d}$. Subsequently the mixture was allowed to cool down to room temperature and diluted with distilled water $(30 \mathrm{~mL})$. The aqueous layer was extracted with $\mathrm{CH}_{2} \mathrm{Cl}_{2}(3 \times 50 \mathrm{~mL})$ and the combined organic phases were washed with distilled water $(75 \mathrm{~mL})$ and brine $(2 \times 75 \mathrm{~mL})$. After drying over anhydrous $\mathrm{MgSO}_{4}$ and filtration, the solvent was removed in vacuo. Silica gel column chromatography ( $n$-pentane $\left./ \mathrm{Et}_{2} \mathrm{O}=40 / 1\right)$ and subsequent recrystallization from ethanol were utilized to purify the residue and afford $\mathbf{1 1}$ as colorless crystals $(3.67 \mathrm{~g}, 7.27 \mathrm{mmol}, 58 \%)$.

TLC: $R \mathrm{f}=0.23$ (silica gel, $n$-pentane: $\mathrm{Et}_{2} \mathrm{O}=40: 1$, [UV]).

${ }^{1} \mathrm{H}$ NMR $\left(500 \mathrm{MHz}, \mathrm{CDCl}_{3}, 300 \mathrm{~K}\right): \delta[\mathrm{ppm}]=1.26\left(\mathrm{~s}, 9 \mathrm{H}, \mathrm{C}\left(\mathrm{CH}_{3}\right)_{3}\right), 2.23\left(\mathrm{~s}, 18 \mathrm{H}, \mathrm{CH}_{3}\right), 6.80(\mathrm{~s}, 3 \mathrm{H}$, Trit-H), 6.89 (s, 6H, Trit-H), $7.41\left(\mathrm{~d},{ }^{4} J=2.4 \mathrm{~Hz}, 1 \mathrm{H}, \mathrm{Ar}-\mathrm{H}\right), 7.77\left(\mathrm{~d},{ }^{4} J=2.4 \mathrm{~Hz}, 1 \mathrm{H}, \mathrm{Ar}-\mathrm{H}\right), 9.83$ (s, 1H, CHO), $11.22(\mathrm{~s}, 1 \mathrm{H}$, Ar-OH).

${ }^{13} \mathrm{C} \mathrm{NMR}\left(126 \mathrm{MHz}, \mathrm{CDCl}_{3}, 300 \mathrm{~K}\right): \delta[\mathrm{ppm}]=21.8,31.2,34.3,63.0,120.2,127.3,128.5,128.7,135.4,136.3$, $137.1,141.3,145.2,158.8,196.9$.

LRMS (ESI, MeCN): $\mathrm{m} / \mathrm{z}=527.4\left[(\mathrm{M}+\mathrm{Na})^{+}\right]$.

EA [\%]: calculated: C 85.67, H 7.99; found: C 85.73, H 8.17.

\section{4-tert-Butyl-2-hydroxymethyl-6-(tris(3,5-dimethylphenyl)methyl)phenol (11)}



$\mathrm{C}_{36} \mathrm{H}_{42} \mathrm{O}_{2}$ $506.72 \mathrm{~g} / \mathrm{mol}$

To a suspension of $\mathbf{1 0}(2.60 \mathrm{~g}, 5.15 \mathrm{mmol}, 1.0 \mathrm{eq})$ in methanol $(100 \mathrm{~mL})$ was added sodium borohydride (390 mg, $10.3 \mathrm{mmol}, 2.0 \mathrm{eq}$ ) in small portions. After the reaction mixture was stirred at room temperature for $2 \mathrm{~h}$, the solvent was removed under reduced pressure. The residue was redissolved in water $(125 \mathrm{~mL})$ and neutralized with glacial acetic acid. The aqueous phase was extracted with chloroform $(3 \times 75 \mathrm{~mL})$ and the combined organic layers were dried over anhydrous $\mathrm{MgSO}_{4}$. After filtration, the solvent was removed in vacuo to yield 12 as a white powder $(2.58 \mathrm{~g}, 5.09 \mathrm{mmol}, 99 \%)$. The product was utilized in the next step without further purification.

${ }^{1} \mathrm{H}$ NMR $\left(500 \mathrm{MHz}, \mathrm{CDCl}_{3}, 300 \mathrm{~K}\right): \delta[\mathrm{ppm}]=1.19\left(\mathrm{~s}, 9 \mathrm{H}, \mathrm{C}\left(\mathrm{CH}_{3}\right)_{3}\right), 2.24\left(\mathrm{~s}, 18 \mathrm{H}, \mathrm{CH}_{3}\right)$, $4.63\left(\mathrm{~s}, 2 \mathrm{H}, \mathrm{CH}_{2}\right), 6.80(\mathrm{~s}, 6 \mathrm{H}$, Trit- $\mathrm{H}), 6.87(\mathrm{~s}, 3 \mathrm{H}$, Trit- $\mathrm{H}), 7.15\left(\mathrm{~d},{ }^{4} \mathrm{~J}=2.5 \mathrm{~Hz}, 1 \mathrm{H}, \mathrm{Ar}-\right.$ $\mathrm{H}), 7.18\left(\mathrm{~d},{ }^{4} J=2.5 \mathrm{~Hz}, 1 \mathrm{H}, \mathrm{Ar}-\mathrm{H}\right)$.

${ }^{13} \mathrm{C}$ NMR $\left(126 \mathrm{MHz}, \mathrm{CDCl}_{3}, 300 \mathrm{~K}\right): \delta[\mathrm{ppm}]=21.8,31.6,34.4,62.7,63.6,124.7,128.3,128.9,132.9,136.3$, $137.0,142.0,144.5,145.2,150.8$.

LRMS (ESI, MeCN): $\mathrm{m} / \mathrm{z}=529.4\left[(\mathrm{M}+\mathrm{Na})^{+}\right]$.

EA [\%]: calculated: C 85.33, H 8.35; found: C 85.04, H 8.99.

\section{2-Bromomethyl-4-tert-butyl-6-(tris(3,5-dimethylphenyl)methyl)phenol (12)}

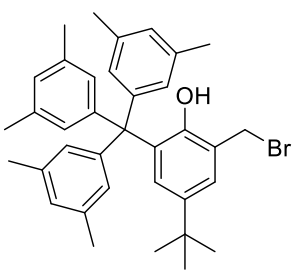

$\mathrm{C}_{36} \mathrm{H}_{41} \mathrm{BrO}$ $569.91 \mathrm{~g} / \mathrm{mol}$

To a solution of 11 (2.58 g, $5.09 \mathrm{mmol}, 1.0$ equiv.) in chloroform (100 mL) was added phosphorus tribromide $(0.24 \mathrm{~mL}, 689 \mathrm{mg}, 2.55 \mathrm{mmol}, 0.5 \mathrm{eq})$. After the reaction mixture was stirred at room temperature for $1 \mathrm{~h}$, iced water $(40 \mathrm{~mL})$ was added over $5 \mathrm{~min}$. The resulting phases were separated and the aqueous phase was extracted with chloroform $(3 \times 25 \mathrm{~mL})$. The combined organic layers were dried over anhydrous $\mathrm{MgSO}_{4}$, filtered and the solvent removed under reduced pressure. The residue was recrystallized from acetonitrile to afford $\mathbf{1 3}$ as colorless crystals $(2.60 \mathrm{~g}, 4.56 \mathrm{mmol}, 90 \%)$.

${ }^{1} \mathrm{H}$ NMR $\left(500 \mathrm{MHz}, \mathrm{CDCl}_{3}, 300 \mathrm{~K}\right): \delta[\mathrm{ppm}]=1.18\left(\mathrm{~s}, 9 \mathrm{H}, \mathrm{C}\left(\mathrm{CH}_{3}\right)_{3}\right), 2.24\left(\mathrm{~s}, 18 \mathrm{H}, \mathrm{CH}_{3}\right)$, $4.52\left(\mathrm{~s}, 2 \mathrm{H}, \mathrm{CH}_{2}\right), 6.78\left(\mathrm{~s}, 6 \mathrm{H}\right.$, Trit-H), $6.88\left(\mathrm{~s}, 3 \mathrm{H}\right.$, Trit-H), $7.14\left(\mathrm{~d},{ }^{4} J=2.4 \mathrm{~Hz}, 1 \mathrm{H}, \mathrm{Ar}-\mathrm{H}\right)$, $7.23\left(\mathrm{~d},{ }^{4} J=2.5 \mathrm{~Hz}, 1 \mathrm{H}, \mathrm{Ar}-\mathrm{H}\right)$.

${ }^{13} \mathrm{C}$ NMR $\left(126 \mathrm{MHz}, \mathrm{CDCl}_{3}, 300 \mathrm{~K}\right): \delta[\mathrm{ppm}]=21.8,31.5,31.5,34.3,62.8,125.1,126.2,128.4,129.0,129.4$, 133.4, 137.1, 142.1, 144.1, 151.0.

LRMS (ESI, MeCN): $\mathrm{m} / \mathrm{z}=593.2\left[(\mathrm{M}+\mathrm{Na})^{+}\right]$.

EA [\%]: calculated: C 75.91, H 7.26; found: C 76.08, H 7.28. 


\section{Kinetic Investigations}
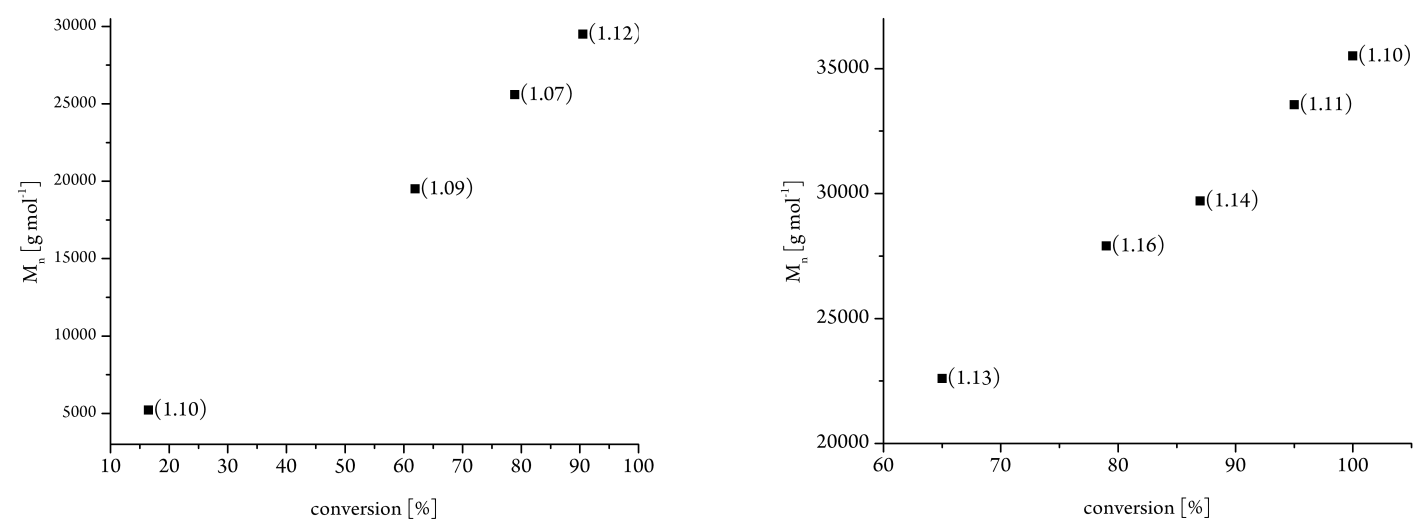

Figure S1. Linear growth of the absolute molecular weight $\left(M_{\mathrm{n}}\right)$ as a function of monomer conversion (determined gravimetically) and the corresponding PDIs. Left: catalyst 1, right: catalyst $\mathbf{2}$. 


\section{Microstructure Analysis}

\section{$3.1 \quad{ }^{13} \mathrm{C}$ NMR spectra}

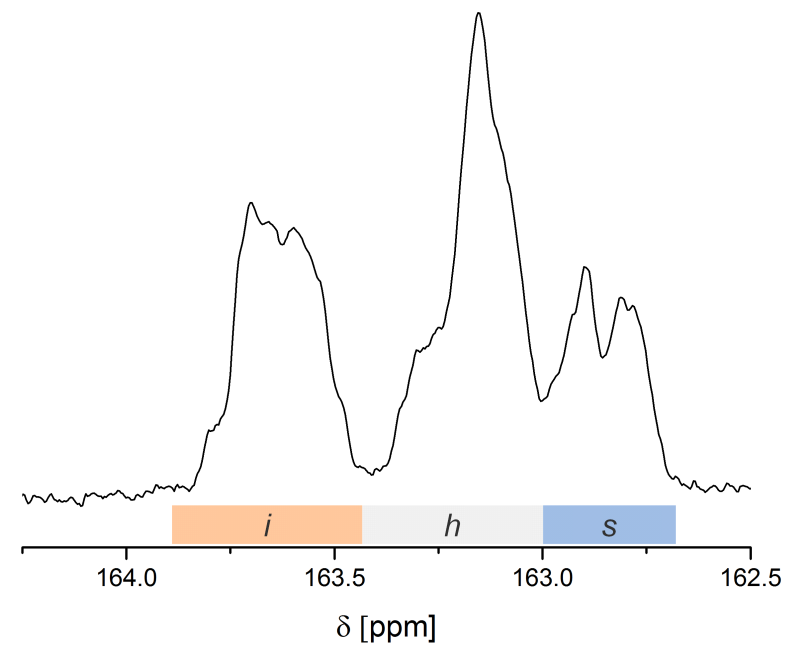

Figure S2. Aromatic quaternary ${ }^{13} \mathrm{C}$ NMR resonances $(i, h, s$ proportions for isotactic, heterotactic, and syndiotactic triads) of poly(2-vinylpyridine) (30 mg of $\mathrm{P} 2 \mathrm{VP}$ in $0.6 \mathrm{~mL}$ of $\mathrm{CD}_{3} \mathrm{OD}$ for catalyst 5 . ([2VP]:[Cat.] $=200: 1,[\mathrm{M}]=2.7 \mathrm{mmol}, 2 \mathrm{~mL}$ of toluene, $\left.25^{\circ} \mathrm{C}\right)$ ).

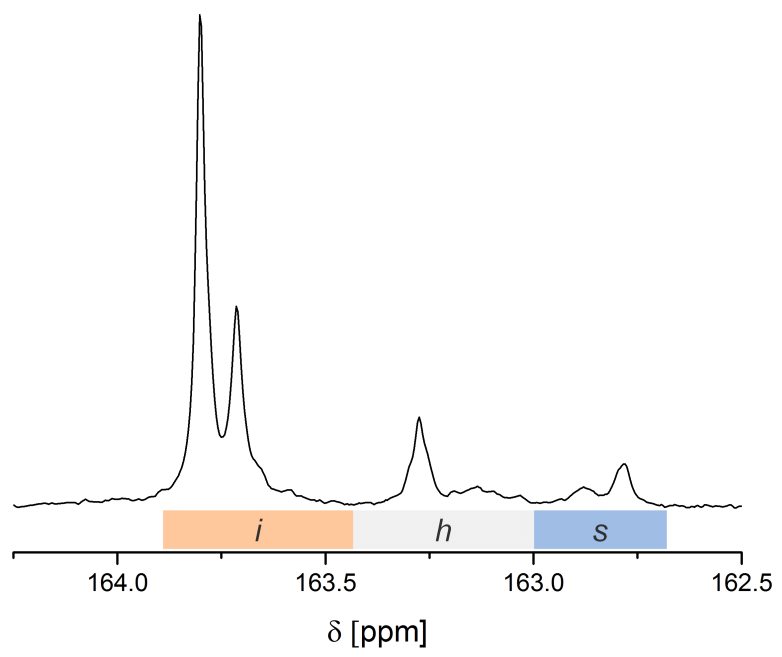

Figure S3. Aromatic quaternary ${ }^{13} \mathrm{C}$ NMR resonances $(i, h, s$ proportions for isotactic, heterotactic, and syndiotactic triads) of poly(2-vinylpyridine) (30 mg of $\mathrm{P} 2 \mathrm{VP}$ in $0.6 \mathrm{~mL}$ of $\mathrm{CD}_{3} \mathrm{OD}$ for catalyst 1. $\left([2 \mathrm{VP}]:[\right.$ Cat. $]=200: 1,[\mathrm{M}]=2.7 \mathrm{mmol}, 2 \mathrm{~mL}$ of toluene, $\left.25^{\circ} \mathrm{C}\right)$ ). 


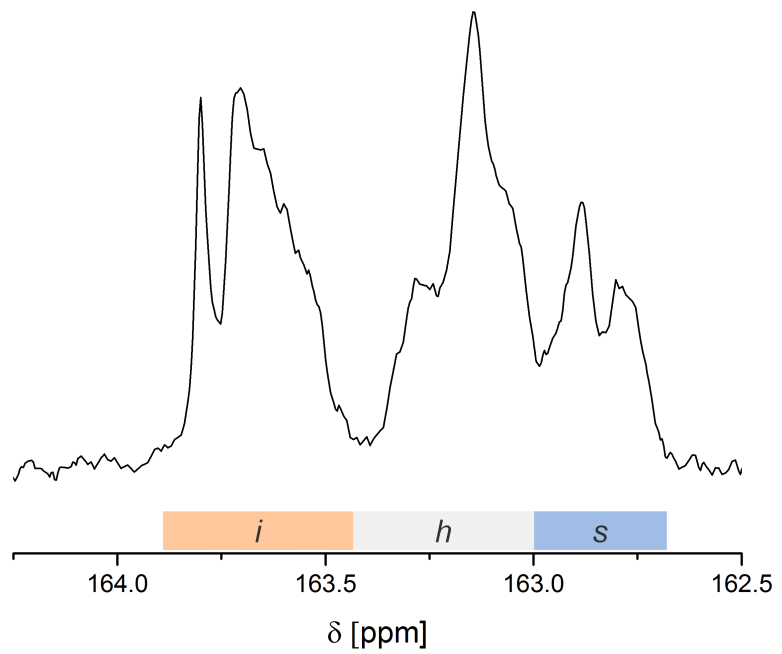

Figure S4. Aromatic quaternary ${ }^{13} \mathrm{C}$ NMR resonances $(i, h, s$ proportions for isotactic, heterotactic, and syndiotactic triads) of poly(2-vinylpyridine) $\left(30 \mathrm{mg}\right.$ of $\mathrm{P} 2 \mathrm{VP}$ in $0.6 \mathrm{~mL}$ of $\mathrm{CD}_{3} \mathrm{OD}$ for catalyst 2. ([2VP]:[Cat.] $=200: 1,[\mathrm{M}]=2.7 \mathrm{mmol}, 2 \mathrm{~mL}$ of toluene, $\left.25^{\circ} \mathrm{C}\right)$ ).



Figure S5. Aromatic quaternary ${ }^{13} \mathrm{C}$ NMR resonances $(i, h, s$ proportions for isotactic, heterotactic, and syndiotactic triads) of poly(2-vinylpyridine) (30 mg of $\mathrm{P} 2 \mathrm{VP}$ in $0.6 \mathrm{~mL}$ of $\mathrm{CD}_{3} \mathrm{OD}$ for catalyst 3. ([2VP]:[Cat.] $=200: 1,[\mathrm{M}]=2.7 \mathrm{mmol}, 2 \mathrm{~mL}$ of toluene, $\left.25^{\circ} \mathrm{C}\right)$ ). 


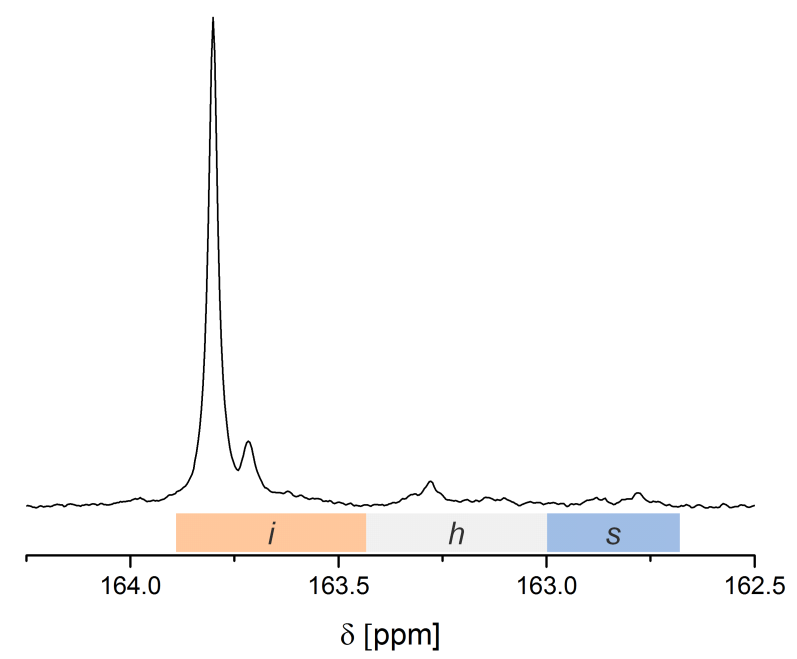

Figure S6. Aromatic quaternary ${ }^{13} \mathrm{C}$ NMR resonances $(i, h, s$ proportions for isotactic, heterotactic, and syndiotactic triads) of poly(2-vinylpyridine) $\left(30 \mathrm{mg}\right.$ of $\mathrm{P} 2 \mathrm{VP}$ in $0.6 \mathrm{~mL}$ of $\mathrm{CD}_{3} \mathrm{OD}$ for catalyst 4. ([2VP]:[Cat.] $=200: 1,[\mathrm{M}]=2.7 \mathrm{mmol}, 2 \mathrm{~mL}$ of toluene, $\left.25^{\circ} \mathrm{C}\right)$ ).

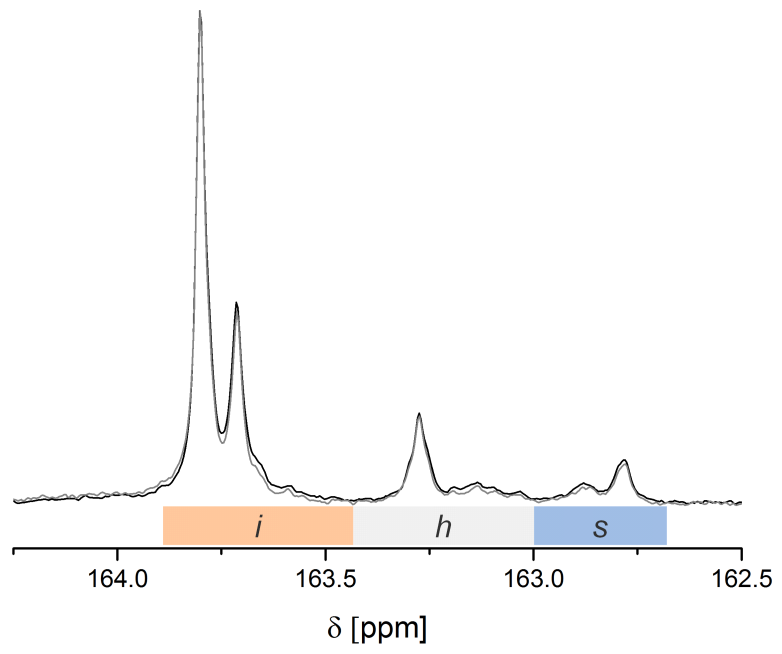

Figure S7. Aromatic quaternary ${ }^{13} \mathrm{C}$ NMR resonances $(i, h, s$ proportions for isotactic, heterotactic, and syndiotactic triads) of poly(2-vinylpyridine) (30 mg P2VP in $0.6 \mathrm{~mL}$ of $\mathrm{CD}_{3} \mathrm{OD}$ for catalysts $\mathbf{1}$. ([2VP]:[Cat.] $=200: 1,[\mathrm{M}]=2.7 \mathrm{mmol}, 2 \mathrm{~mL}$ of toluene, $25^{\circ} \mathrm{C}$ : black, $50^{\circ} \mathrm{C}$ : grey)). 


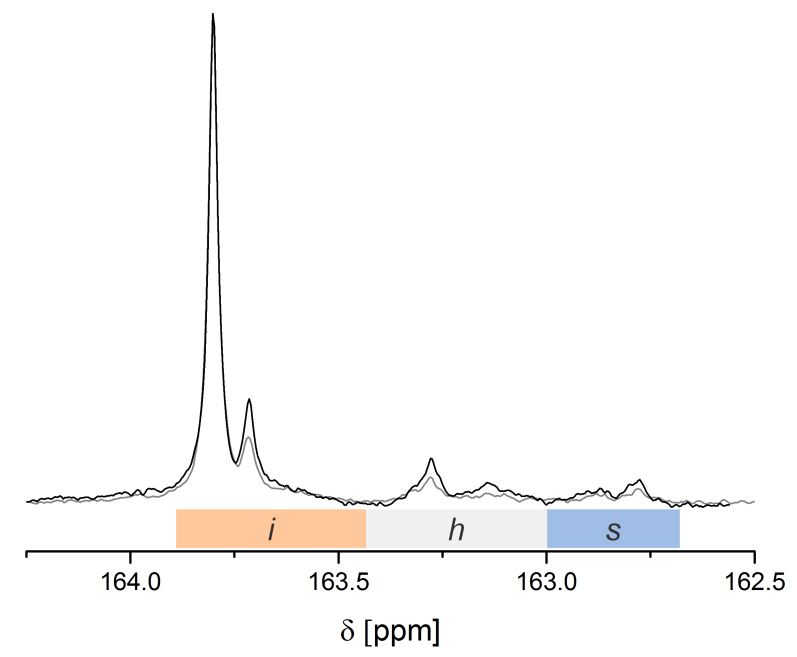

Figure S8. Aromatic quaternary ${ }^{13} \mathrm{C}$ NMR resonances $(i, h, s$ proportions for isotactic, heterotactic, and syndiotactic triads) of poly(2-vinylpyridine) $\left(30 \mathrm{mg} \mathrm{P} 2 \mathrm{VP}\right.$ in $0.6 \mathrm{~mL}$ of $\mathrm{CD}_{3} \mathrm{OD}$ for catalysts 4. ([2VP]:[Cat.] $=200: 1,[\mathrm{M}]=2.7 \mathrm{mmol}, 2 \mathrm{~mL}$ of toluene, $25^{\circ} \mathrm{C}$ : black, $50^{\circ} \mathrm{C}$ : grey)).

\subsection{Theoretical Investigations}

- $\mathrm{P}_{\mathrm{m}}$ is the probability of meso linkages between monomer units and is determined by ${ }^{13} \mathrm{C} \mathrm{NMR}$ spectroscopy.

- Theoretical triad distributions are calculated using the following correlations:

Probability of prochiral monomer addition via $r e$ or $s i$ side of the catalyst, where $P_{m}=m=\sigma+(1-\sigma)^{2}$ and $\mathrm{P}_{\mathrm{r}}=1-\mathrm{P}_{\mathrm{m}}$

$$
\begin{gathered}
\mathrm{mm}=\mathrm{P}_{\mathrm{m}}{ }^{2} \\
\mathrm{mr}=\mathrm{P}_{\mathrm{m}} \mathrm{P}_{\mathrm{r}} \\
\mathrm{rr}=\mathrm{P}_{\mathrm{r}}{ }^{2}
\end{gathered}
$$




\section{Thermoanalysis}

\subsection{DSC}



Figure S9. DSC curves of crystallized P2VP samples at different crystallization temperatures $\mathrm{T}_{\mathrm{c}}$ for $150 \mathrm{~min}$ (catalyst $4,[2 \mathrm{VP}]:[$ Cat. $]=200: 1,[\mathrm{M}]=2.7 \mathrm{mmol}, 2 \mathrm{~mL}$ toluene, $25^{\circ} \mathrm{C}$ ).

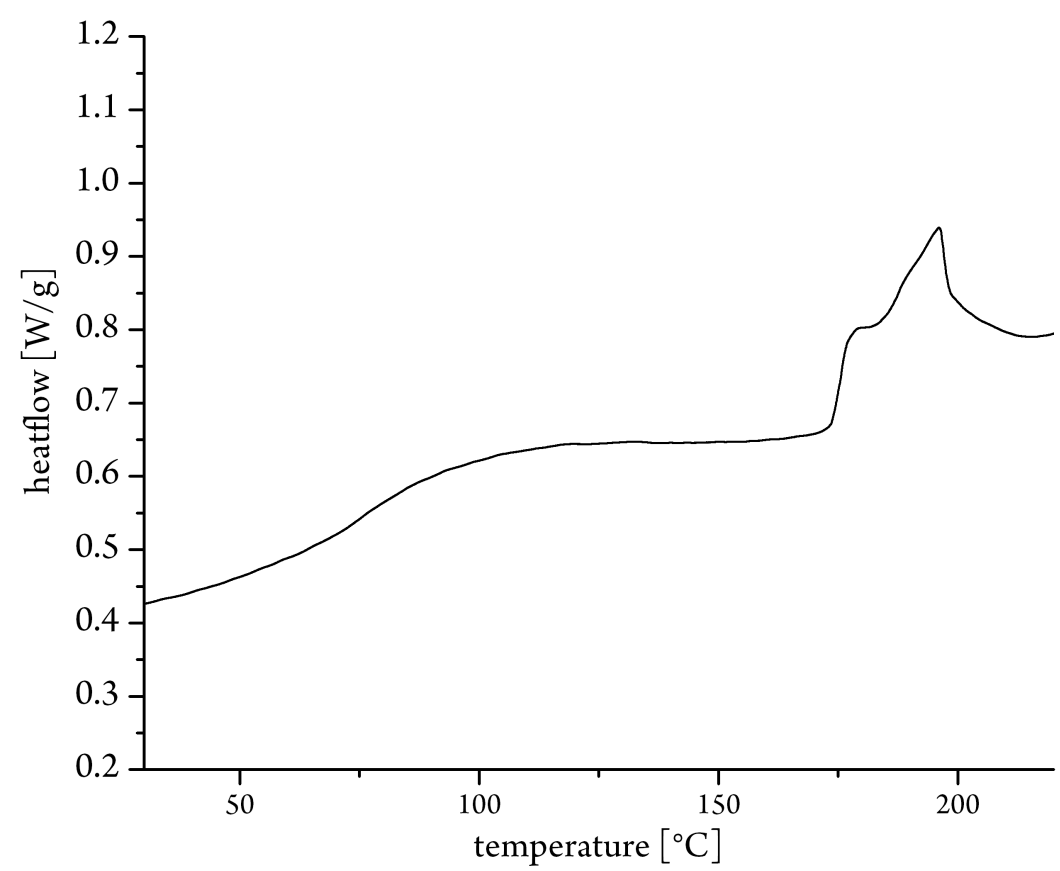

Figure S10. DSC curves of crystallized P2VP samples at a $\mathrm{T}_{\mathrm{c}}=130^{\circ} \mathrm{C}$ for $16 \mathrm{~h}$ (catalyst 1, $[2 \mathrm{VP}]:[$ Cat. $]=200: 1,[\mathrm{M}]=2.7 \mathrm{mmol}, 2 \mathrm{~mL}$ of toluene, $\left.25^{\circ} \mathrm{C}\right)$. 


\subsection{TGA}
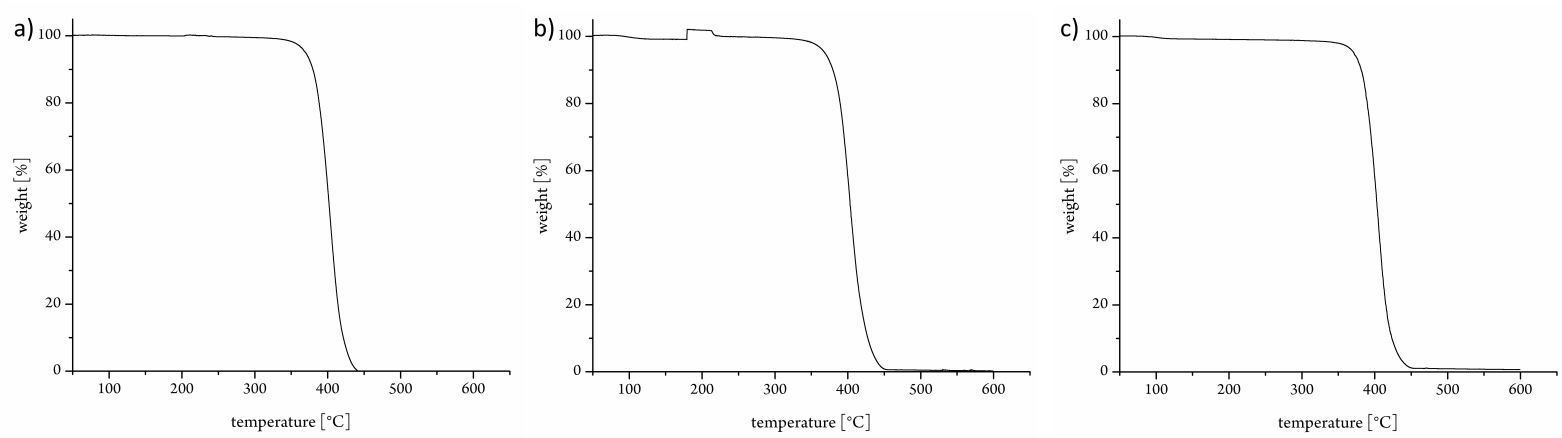

Figure S11. TGA curves of atactic (a), highly isotactic amorphous $\left(\mathrm{P}_{\mathrm{m}}=0.92, \mathrm{~b}\right)$, and highly isotactic crystallized P2VP $\left(\mathrm{P}_{\mathrm{m}}=0.92, \mathrm{~T}_{\mathrm{c}}=170{ }^{\circ} \mathrm{C}\right.$, crystallization time $\left.150 \mathrm{~min}, \mathrm{c}\right)$. 


\section{Literature}

[1] K. C. Hultzsch, P. Voth, K. Beckerle, T. P. Spaniol, J. Okuda, Organometallics 2000, 19, 228-243.

[2] P. T. Altenbuchner, F. Adams, A. Kronast, E. Herdtweck, A. Pöthig, B. Rieger, Polym. Chem. 2015, 6, 6796-6801. 